\title{
Artificial living crystals in confined environment
}

\author{
Wen Yang, ${ }^{1,2}$ Vyacheslav R. Misko, ${ }^{2,3}$ Jacques Tempere, ${ }^{3,4}$ Minghui Kong, ${ }^{5}$ and Francois M. Peeters ${ }^{2,6}$ \\ ${ }^{1}$ College of Materials Science and Engineering, Taiyuan University of Science and Technology, Taiyuan 030024, People's Republic of China \\ ${ }^{2}$ Departement Fysica, Universiteit Antwerpen, Groenenborgerlaan 171, B-2020 Antwerpen, Belgium \\ ${ }^{3}$ TQC, Universiteit Antwerpen, Universiteitsplein 1, B-2610 Antwerpen, Belgium \\ ${ }^{4}$ Lyman Laboratory of Physics, Harvard University, Cambridge, Massachusetts 02138, USA \\ ${ }^{5}$ Institute of Plasma Physics, Chinese Academy of Sciences, Hefei 230031, People's Republic of China \\ ${ }^{6}$ Departamento de Física, Universidade Federal do Ceará, Caixa Postal 6030, Campus do Pici, 60455-760 Fortaleza, Ceará, Brazil
}

(Received 11 July 2016; revised manuscript received 1 April 2017; published 5 June 2017)

\begin{abstract}
Similar to the spontaneous formation of colonies of bacteria, flocks of birds, or schools of fish, "living crystals" can be formed by artificial self-propelled particles such as Janus colloids. Unlike usual solids, these "crystals" are far from thermodynamic equilibrium. They fluctuate in time forming a crystalline structure, breaking apart and re-forming again. We propose a method to stabilize living crystals by applying a weak confinement potential that does not suppress the ability of the particles to perform self-propelled motion, but it stabilizes the structure and shape of the dynamical clusters. This gives rise to such configurations of living crystals as "living shells" formed by Janus colloids. Moreover, the shape of the stable living clusters can be controlled by tuning the potential strength. Our proposal can be verified experimentally with either artificial microswimmers such as Janus colloids, or with living active matter.
\end{abstract}

DOI: 10.1103/PhysRevE.95.062602

\section{INTRODUCTION}

The dynamics of self-propelled (SP) particles, also called microswimmers, has become a forefront theme in biophysics research since the last decade $[1,2]$. Collections of microswimmers were studied as a physical model to understand complex behaviors of a wide range of active matter including biological and artificial systems [3]. Typical biological active systems are usually associated with various living swimmers, such as bacteria like Escherichia coli [4], spermatozoa [5], volvox [6], etc., while artificial active systems were inspired by biological swimmers and were employed by alternative concepts to consume chemical reaction energy or heat to generate directed motion of the microswimmers. In this sense, artificial microswimmers are potentially more efficient. Known synthetic microswimmers include Janus colloids [7,8], bimetallic microspheres [9], rotating discs [10], etc. These active systems have potential applications in medicine, biology, material, and environmental science $[2,3,11]$.

Previous works on microswimmers have exhibited a range of exotic behaviors compared to those of passive systems. These mainly comprise the dynamics of the directed motion $[12,13]$, motion rectification in patterned environment [14-20], giant density fluctuations [21-23], dynamic phase transitions [24-27], and motility-sorting behavior in a microchannel [28-30]. While the literature on microswimmers increased dramatically in recent years, some basic questions concerning the equilibrium and nonequilibrium statistics remained open, such as their phase behavior.

Recent experiments [31,32] reported on the formation of a "living crystal" when the phase behavior of the microswimmers can be monitored by external driving light. In those experiments, the microswimmers aggregated into a "crystal" when the light was on while the crystal dissolved when the light was off. Such a crystal was called "living crystal" or "dynamical cluster" as it was nonequilibrium in nature. The crystal could form, break, explode, and reform elsewhere being illuminated by light [31,32]. In this respect, an important question arises: Would it be possible to stabilize the living crystal, preventing it from sudden fluctuation-driven evolution? To address this question, here we propose a simple model system: self-propelled particles in a trap. Without loss of generality, the trap is chosen parabolic. The trap is weak such that the self-propelled particles are able to move freely and aggregate in clusters. The strength of the confinement is chosen to prevent moderate fluctuations of the shape and the average number of particles in the cluster. In this way, we were able to obtain dynamical clusters of regular and well-controlled shapes (although thermodynamically nonequilibrium) which did not evolve in time (i.e., not breaking, exploding, reforming, etc.). Such clusters in the shape of a ring are dynamically stable structures of living crystals. Our analysis shows that both the shape (also, size) and the microstructure can be monitored by the self-velocity of the microswimmers which is achieved by tuning the intensity of the external driving light in the experiment. Note that in the previous studies of dynamical clusters $[26,27,33,34]$, the authors addressed the collective behavior of microswimmers but did not discuss the microstructure of their aggregates. In this work, we analyze the collective properties and the new stable phases as well as the microstructure of the clusters formed by microswimmers in the presence of an attractive potential.

The paper is organized as follows. First, the model and the numerical approach are described in Sec. II. Then, in Sec. III, we discuss various phases of the dynamical clusters in the trap depending on the density of the microswimmers, i.e., formation of a lattice, elastically deformed lattice and the saturated state. Next, in Sec. IV, we analyze the microstructure of the dynamical clusters including shells and close-packed circular clusters, and the resulting phase diagram of the microswimmers. The numerical simulations of the phases are supported by analytical results based on a single-particle model. Finally, our conclusions are presented in Sec. V. 


\section{SIMULATION}

In our model, $N$ microswimmers (MS) (also called SP particles) move in a $L \times L$ simulation box where a parabolic confinement potential of diameter $D$ is located at the center of the square. Periodic boundary conditions are applied in the $x$ and $y$ directions. Following the numerical model of Refs. [14,23], the SP particles are considered as soft disks with diameter $d$ interacting through a repulsive short-range force. The motion of the microswimmers is described by the overdamped Langevin-type equation:

$$
\frac{\partial \mathbf{r}_{i}}{\partial t}=v_{0} \hat{v}_{i}+\mu\left(\sum_{j \neq i} \mathbf{F}_{i j}+\mathbf{F}_{i}^{p}\right),
$$

where $v_{0}$ is the self-propulsion velocity, and $\mu$ is the mobility of the microswimmers. The repulsive interaction force between particle $i$ and particle $j$ is $\mathbf{F}_{i j}=\kappa \alpha_{i j} \hat{\mathbf{r}}_{i j}$, if $\alpha_{i j}>0\left(\mathbf{F}_{i j}=0\right.$ otherwise), $\alpha_{i j}=1 / 2\left(d_{i}+d_{j}\right)-r_{i j}$ is the overlap distance between particle $i$ and $j$, and $r_{i j}$ is the distance between particle $i$ and $j$. The force due to the potential is $\mathbf{F}_{i}^{p}=-A \mathbf{r}$, if $r<$ $D / 2\left(\mathbf{F}_{i}^{p}=0\right.$ otherwise $)$ is directed to the center of the trap with the strength of the potential $A>0$. The stochastic term $\hat{v}_{i}=\left(\cos \theta_{i}, \sin \theta_{i}\right)$ represents a random-direction self-velocity of particle $i$, with $\theta_{i}(t)$ proportional to a Gaussian white noise $\eta_{i}(t)$ :

$$
\begin{gathered}
\frac{\partial \theta_{i}}{\partial t}=\eta_{i}(t), \\
\left\langle\eta_{i}(t)\right\rangle=0, \quad\left\langle\eta_{i}(t) \eta_{j}\left(t^{\prime}\right)\right\rangle=2 D_{r} \delta_{i j} \delta\left(t-t^{\prime}\right) .
\end{gathered}
$$

Here $D_{r}$ is the rotational diffusion coefficient of the microswimmers, or the angular noise. Since the effect of thermal noise has been studied elsewhere [14], here we neglect thermal noise to emphasize the effect of rotational noise [23].

It is useful to make a transformation to dimensionless units. For this purpose, we chose the MS diameter $d$ as the unit of length $d \equiv r_{0}$ and $t_{0}=1 /\left(\mu_{0} \kappa_{0}\right)$ as the unit of time. Then typical simulation parameters are as follows: $L=100$, $D=30, d=1, \kappa=10, \mu=1$, and the total simulation time $t_{e}=4000$ with a time step $\Delta t=0.001$. The total simulation time $t_{e}$ should be sufficient for the system to achieve the dynamical equilibrium state. Clearly, this time depends on the number of particles in the system and the location of the trajectories of the moving particles, i.e., whether they are inside or outside the trap. To find the optimal time, we performed numerous simulation tests. Since we are interested in the dynamical equilibrium states inside the trap, an important indication of achieving dynamical equilibrium is the total number of particles in the trap. Figure 1 shows the number of microswimmers inside the trap versus simulation time $t$ of the system with the total number of SP particles varied in a broad range from $N=100$ to 12500 . As seen from the plot, the number of SP particles in the trap increases gradually to the maximum number at about $t \approx 1000$ and this number saturates at $t \approx 2000$. It is also clear from the plot that for $N \lesssim 1000$, all the SP are trapped. For a larger total number of SP particles, i.e., $N>1000$, the saturation of the number of trapped particles occurs for even shorter times, $t<1000$. However, now the number of trapped particles

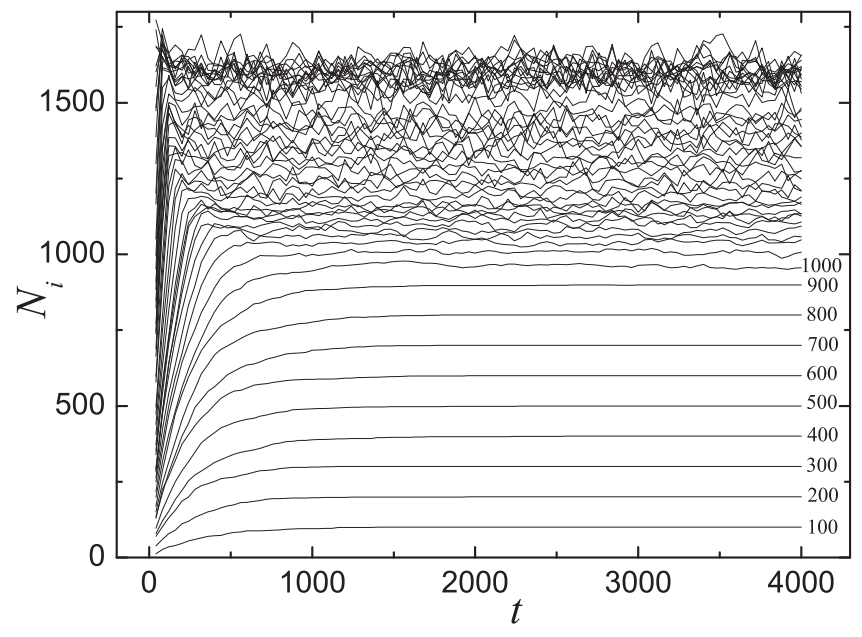

FIG. 1. The number of microswimmers in the trap $N_{i}$ versus simulation time $t$ of systems with the total particle number varied from $N=100$ to 12500 , for the rotational diffusion coefficient $D_{r}=0.005$, the self-propulsion velocity $v_{0}=1.0$, and the maximum potential strength $A=0.1$. The step value of total number $N$ has been chosen as follows: $\Delta N=100$ for $N<2000 ; \Delta N=200$ for $N=2000-5000$; and $\Delta N=500$ for $N>5000$. The total number $N$ (when $N \leqslant 1000$ ) is shown in the plot for the corresponding curves.

fluctuates around some average value (which is less than the total number of SP particles in the system). This means that the system of microswimmers reached the dynamical equilibrium state when the numbers of microswimmers entering the trap and leaving it are in balance. In our simulations, the numbers of SP in the trap were calculated as averaged values over the time interval $2000<t \leqslant 4000$.

\section{MS PHASES IN THE TRAP: LATTICE, DEFORMED LATTICE, AND SATURATED STATE}

As shown in Fig. 1, due to the finite size of the trap, it cannot accommodate more than $N_{i} \approx 1600$ particles for any total number of particles up to $N=12500$. This is illustrated more clearly in Fig. 2 where we show the number of particles in the trap $N_{i}$ versus the total number of particles $N$ in our computational unit cell.

The curve in the main panel of Fig. 2 contains two distinct linear parts. The first linear part (for $0<N<1000$ ) has a slope of 1.0, i.e., all the microswimmers, become trapped and form a regular hexagonal lattice (indicated as phase "I" in Fig. 2). This phase corresponds to the smooth $N_{i}(t)$ curves in Fig. 1. For the parameters used in our simulations, the area of the parabolic trap is 900 times the area of a single microswimmer: $\pi \times 15^{2}=900 \times \pi \times 0.5^{2}$. When the entire trap is filled by the microswimmers (arranged in a hexagonal lattice), and additional microswimmers try to enter the trap from outside, they produce elastic deformations. The elastic deformation energy is compensated by the pressure produced by microswimmers moving outside the trap (called "swim pressure" $[35,36])$. Thus, not all the MSs can now be trapped. The constant slope of the $N_{i}(N)$ curve (which is 0.12 for $1000<N<6000$ ) is explained by the elastic response of the microswimmers which are soft disks. With increasing the total number of microswimmers, the swim pressure produced by 


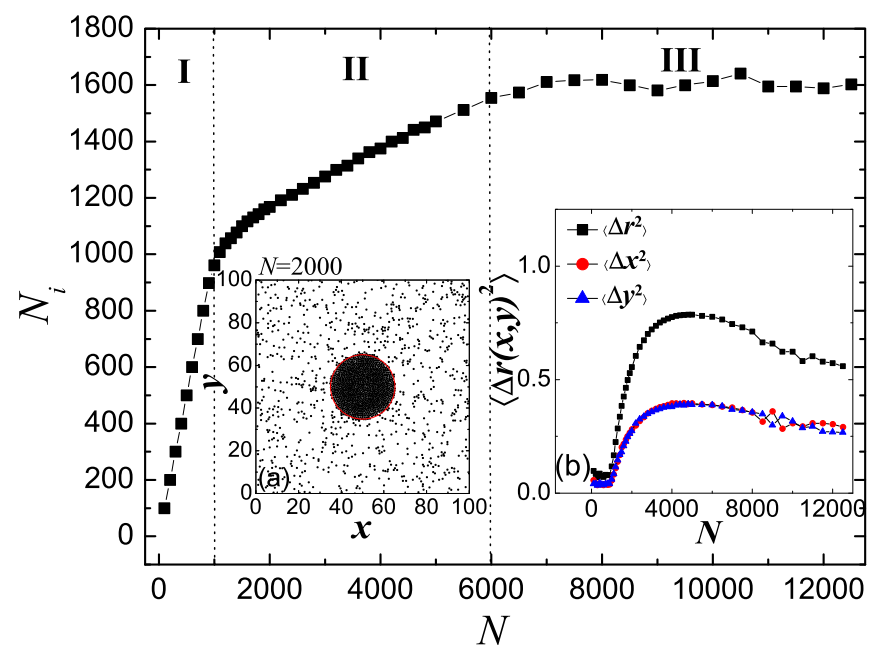

FIG. 2. The number of microswimmers in the trap $N_{i}$ versus the total particle number $N$ varying from $N=100$ to 12500 , for $D_{r}=$ $0.005, v_{0}=1.0, A=0.1$. The step value of $N$ is the same as in Fig. 1. Regions labeled with I, II, and III correspond to "lattice," "elastically deformed lattice," and "saturated" state. Inset (a): Snapshot of the microswimmers for $N=2000$, where the parabolic trap is shown by the red circle. Inset (b): The mean square displacement (MSD) of the microswimmers versus the total particle number $N$, in the $x y$ plane (squares); the $x$ and $y$ components of the MSD (circles and triangles, respectively).

the particles moving outside the trap further increases until the number of MSs inside the trap saturates at about $N_{i}=1600$. This is achieved for the total number of MSs $N \approx 6000$ (see Fig. 2). This second critical point (i.e., when $N_{i}$ saturates) is related to the onset of aggregation of MSs outside the trap. The clusters, which gradually percolate with increasing $N$ (this will be discussed below), possess the same elastic properties as the trapped MSs, and this solidification of the phase outside the trap occurs at a constant swim pressure until the MSs fill all the space, inside and outside the trap. This "saturated" regime is extended from $N=6000$ to 12500 (the maximum number of particles per simulation cell used in our simulations) and is characterized by a constant, weakly fluctuating, number of trapped particles $N_{i} \approx 1600$.

To analyze self-diffusion of the microswimmers, we calculated the mean square displacement (MSD) of the system, which is presented as inset (b) of Fig. 2. Each point of the MSD curves is obtained by averaging over time. It is clearly shown that the MSD of the system with $N<1000$ is close to zero because all the microswimmers are trapped. The fast increase of the MSD for $N \gtrsim 1000$ is related to the appearance of free microswimmers in the system; then the MSD remains increasing while the total number of microswimmers increases within the region $1000<N<5000$. Finally, the MSD saturates at about $N \approx 5000$ and starts to decrease since the mobility of the particles gradually decreases due to their aggregation in clusters outside the trap.

\section{THE STRUCTURE OF THE CLUSTERS IN THE TRAP}

Above, we analyzed various regimes (shown in Fig. 2) of filling the trap by microswimmers. Here we investigate the internal structure of the formed clusters in the trap.

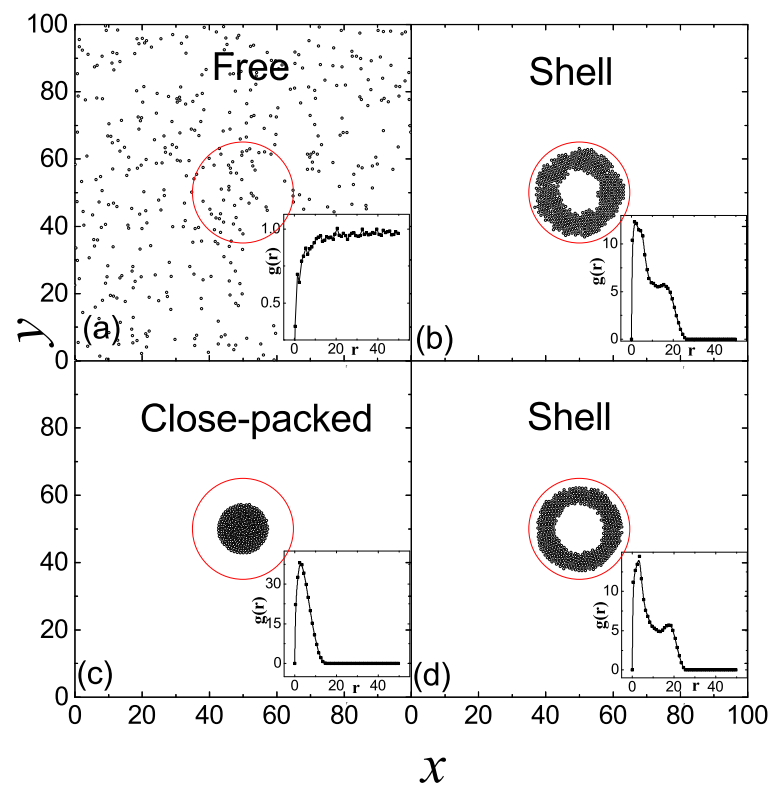

FIG. 3. (a)-(d) Structures formed by $N=500$ microswimmers for varying parameters: (a) $D_{r}=0.005, v_{0}=1.0, A=0.05$; (b) $D_{r}=0.005, v_{0}=1.0, A=0.1$; (c) $D_{r}=0.005, v_{0}=1.0, A=$ 0.5 ; (d) $D_{r}=0.005, v_{0}=5.0, A=0.5$. Insets in (a)-(d) are the corresponding radial distribution functions $[g(r)]$.

Typical snapshots of the clusters formed by $N=500$ microswimmers are presented in Figs. 3(a)-3(d). The insets show the corresponding radial distribution function (RDF) normalized to that of a random system of particles. The systems in the upper panels [(a) and (b)] differ by the trap strength $A$. The lower panels [(c) and (d)] show the distributions for varying self-propelled velocity $v_{0}$.

Figure 3(a) shows a snapshot of the self-propelled microswimmers when the trap (shown by the red circle at the center) is very weak $(A=0.05)$. The motion of the MSs is practically not influenced by the trap, and we can call this phase "free." The related RDF is flat approaching the value of 1.0 which indicates that the motion of MSs is random. Increasing the strength of the trap to $A=0.1$ results in a striking self-organization of the microswimmers in a shell structure near the boundary of the trap [Fig. 3(b)]. Note that such a shell structure formed by self-propelled particles is different from the structure of a cluster of passive particles in a trap, which is naturally close-packed [37]. Also, this new shell structure is different from clusters of self-propelled particles, or living crystals, found earlier in the absence of a confinement potential which had either near-circular shape [23] or fluctuating irregular shapes [31-33]. The formation of the shell clusters is due to the competition between the confining potential and the self-propulsion velocity. The confinement force is minimum at the center of the trap, and the microswimmers moving with velocity $v_{0}$ avoid the central part but not the boundary where the force due to the confinement is larger. As a result, the filling of the trap by microswimmers occurs from the boundary toward the center. However, this depends on the relation between the parameters of the system. For example, for strong enough confinement and large $v_{0}$, additional particles can preferably leave the trap rather than 
fill it near the center. In this way, we are able to control the width of the shell and the density of particles in the shell.

With further increasing trap strength, the radius of the shell decreases, and for very large strength, i.e., $A=0.5$, the central opening disappears, and the microswimmers form close-packed structures [see Fig. 3(c)] as in the case of confined passive particles. This can be explained as a suppression of the self-propelled motion of the microswimmers in a very strong trap. Indeed, the formation of a shell is due to the balance between the self-propulsion velocity and the trap strength. If the trap is too weak, the shell dissolves. In the opposite case of a very strong trap, the shell collapses to a close-packed cluster. Clearly, increasing the self-propulsion velocity $v_{0}$ (for the same large value of the trap strength $A=0.5$ ) restores the shell structure, as shown in Fig. 3(d). For even larger microswimmer velocity, e.g., $v_{0}=0.8$, the shell dissolves, due to the escape of the microswimmers from the trap, which become free again. These changes are further illustrated by the calculated RDF (normalized to that for a system of free particles) shown in the insets of Figs. 3(a)-3(d). Thus for the free phase [Fig. 3(a)] the RDF saturates at a value equal to 1 . For a wide shell, when microswimmers are organized in a regular lattice, the RDF has a peak and a shoulder [Fig. 3(b)]. The shoulder disappears when the shell is compressed to a dense circular cluster [Fig. 3(c)] and reappears again transforming into a broad peak in the case of a dense shell [Fig. 3(d)].

To summarize, three different regimes are found inside the parabolic trap when varying the trapping potential and the propulsion speed. These numerical results are consistent with the theoretical model developed by Solon and co-workers [38]. According to this model, in an infinite harmonic trap, for a single active particle, Eqs. (1) and (2) can be rewritten as follows:

$$
\begin{gathered}
\frac{\partial r_{i}}{\partial t}=-A r_{i}+v_{0} \cos \theta_{i}, \\
\frac{\partial \theta_{i}}{\partial t}=\sqrt{2 D_{r}} \eta(t)-\frac{v_{0}}{r_{i}} \sin \theta_{i},
\end{gathered}
$$

where the angle $\theta_{i}$ is the direction between the particle and the normal to the trap, and $\eta(t)$ is a Gaussian unit white noise. Using dimensionless variables $\tau=t D_{r}, \tilde{r}_{i}=r_{i} A / v_{0}$, and $\eta(\tau)=\eta(t) / \sqrt{D_{r}}$, the above equations become [38]

$$
\begin{aligned}
\frac{\partial \tilde{r_{i}}}{\partial t} & =-\frac{A}{D_{r}}\left(\tilde{r_{i}}-\cos \theta_{i}\right), \\
\frac{\partial \theta_{i}}{\partial t} & =\sqrt{2} \tilde{\eta}-\frac{A}{\tilde{r_{i} D_{r}}} \sin \theta_{i} .
\end{aligned}
$$

Thus, $\theta_{i}$ undergoes rotational diffusion in an effective potential $\left(A / \tilde{r}_{i} D_{r}\right)\left(1-\cos \theta_{i}\right)$, whose amplitude diverges as $D_{r} / A \rightarrow 0$. In this limit, as shown in Ref. [38], the particle is almost always at the border of the trap, although the fluctuations of $\theta_{i}$ prevent it from reaching exactly $r_{i} \sim v_{0} / A$, in a band of thickness $D_{r} v_{0} / 2 A^{2}$. The above model is fit for an infinite harmonic trap [38]. We adopt this model to treat our case, where the parabolic trap is cut off at a radius of $D / 2$. Therefore, the active particle will be trapped only when $v_{0} / A<D / 2$ and will be untrapped otherwise. This explains
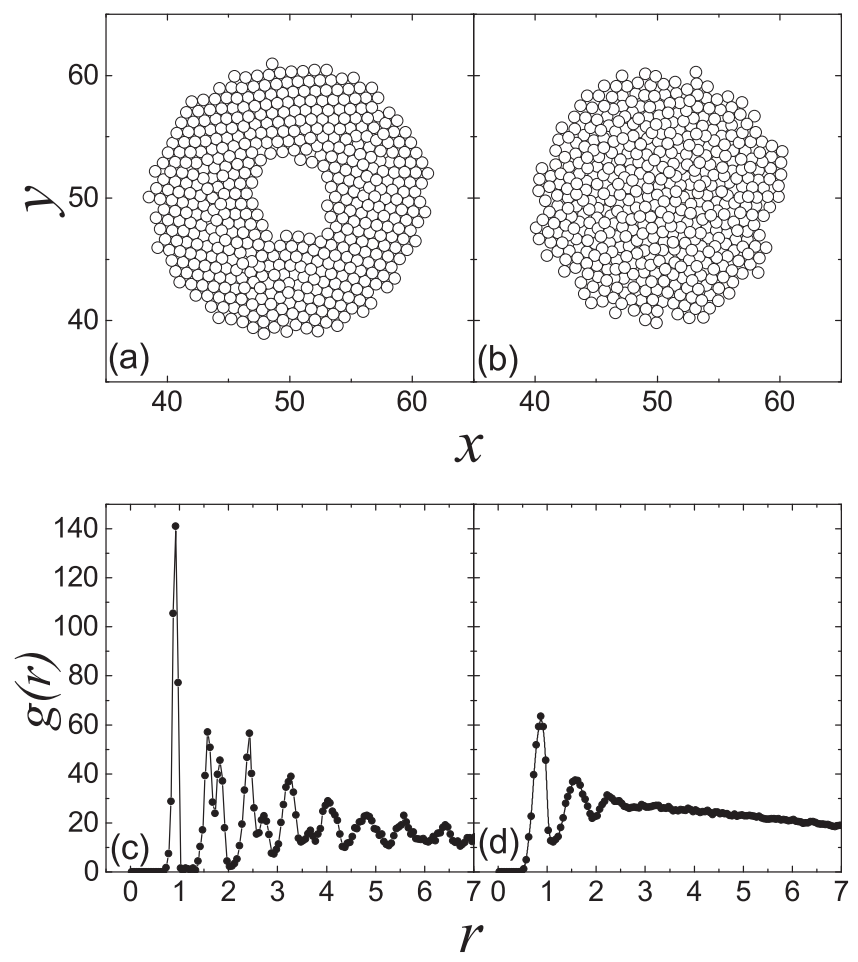

FIG. 4. (a),(b) Structures formed by $N=500$ microswimmers for varying parameters: (a) $D_{r}=0.005, v_{0}=1.5, A=0.2$; and (b) $D_{r}=5.0, v_{0}=1.5, A=0.2$. The corresponding radial distribution functions $g(r)$ are presented in (c) and (d). For the highly ordered structure (a), the function $g(r)$ shows clear peaks indicating strong hexagonal correlations (c). For the case of much higher rotational noise (b), the hexagonal correlations are suppressed (d).

the appearance of the boundary between the free and the shell "phases," as was shown in Fig. 3. Furthermore, the trapping radius $r \sim v_{0} / A$ and band thickness $D_{r} v_{0} / 2 A^{2}$ found in Ref. [38] are consistent with our simulations results, which makes this model useful for the estimation of the trapping area of the active particles in a parabolic potential.

In addition, we analyzed the influence of the rotational noise $D_{r}$ on the structure formed by the microswimmers in the trap. We revealed that the phases (e.g., a shell or close-packed cluster) are rather robust with respect to moderate changes of $D_{r}$. For example, an increase of $D_{r}$ from 0.005 up to 0.5 results only in a change of the local structure of the shell from a regular lattice to a liquidlike structure. However, for $D_{r}=5.0$, the shell dissolves and the particles fill the interior of the trap [cf. Figs. 4(a) and 4(b)]. The effect of noise on the local structure is further analyzed by calculating the corresponding radial distribution function $g(r)$ [note that $g(r)$ characterizes the positional order of the particles but not their orientation]. Under low noise, the microstructures of active particles look crystalline [see Fig. 4(a)], and the corresponding $g(r)$ [Fig. 4(c)] shows many peaks. When the noise is increased, the function $g(r)$ in Fig. 4(d) indicates that the structure is disordered: while short-range hexagonal correlations still remain, the long-range crystalline order is destroyed. This shows that a crystal formed by active particles is not structurally different from a crystal formed by passive 

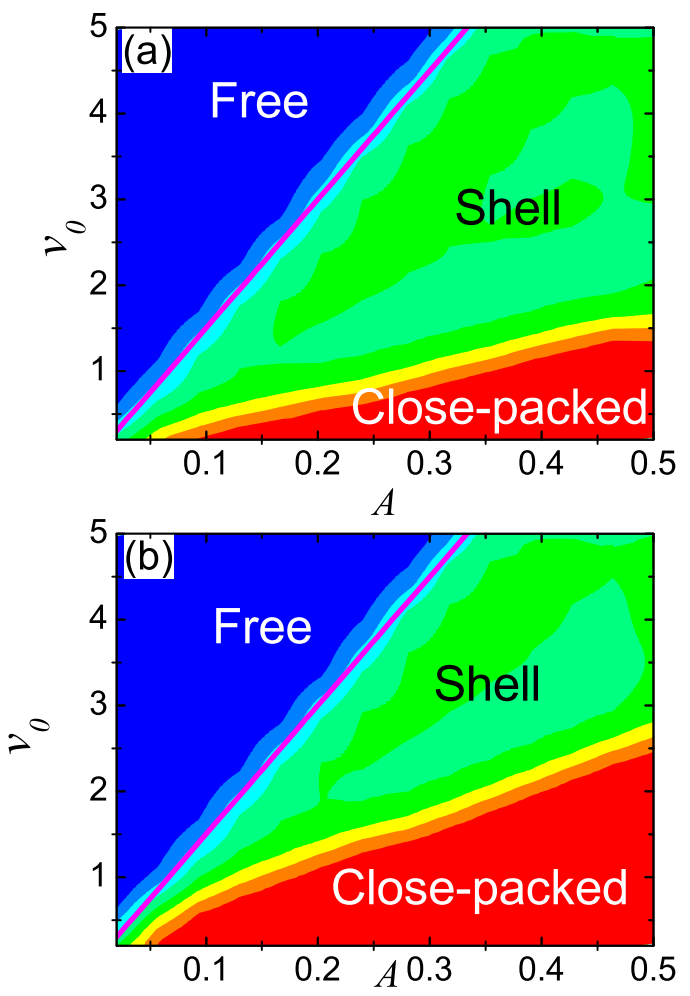

FIG. 5. Phase diagram of the systems in the plane "self-velocity $v_{0}$ versus trap strength $A$," for the total number of particles $N=100$ (a) and $N=500$ (b), for $D_{r}=0.005$ and varying $A$ and $v_{0}$. The phases, i.e., free, shell, and close-packed, are indicated in the plot. The theoretical phase boundary between the free and shell phases, calculated using a single-particle model, $v_{0} / A=D / 2=15$, is shown by a solid magenta line.

colloids, which will also go through dislocation-unbinding transitions as noise increases.

The above analysis of various structures, or phases, is summarized in the phase diagrams in the plane "self-velocity $v_{0}$ versus trap strength $A$ " shown in Fig. 5 for two values of the total number of microswimmers, $N=100$ and $N=$ 500. In the figure, the value of $A$ varies from $A=0.01$ to $A=0.5$ with an interval of $\Delta A=0.05$, and $v_{0}=0.1$ to 5.0 with $\Delta v_{0}=0.5$. We distinguish three phases, namely, free microswimmers (shown by blue color "shell" (green), and "close-packed" cluster (red). The diagrams show that (i) low values of $A$ always result in the free phase; the region becomes broader with increasing $v_{0}$; (ii) low values of $v_{0}$ facilitate the appearance of the close-packed cluster; this region extends when increasing $A$; (iii) the shell structures are observed along the line $v_{0} / A=10$; the region becomes broader when both $v_{0}$ and $A$ increase, moreover, the shell region in the phase diagram squeezes with increasing number of particles in the system; (iv) the boundaries between the phases oscillate reflecting the sequential filling of the single-row shells inside the shell structures and the close-packed circular clusters by microswimmers. For comparison, the theoretical phase boundary between the free and shell phases, calculated using a single-particle model, $v_{0} / A=D / 2=15$, is shown in Fig. 5 . The theoretical phase boundary agrees with the simulated
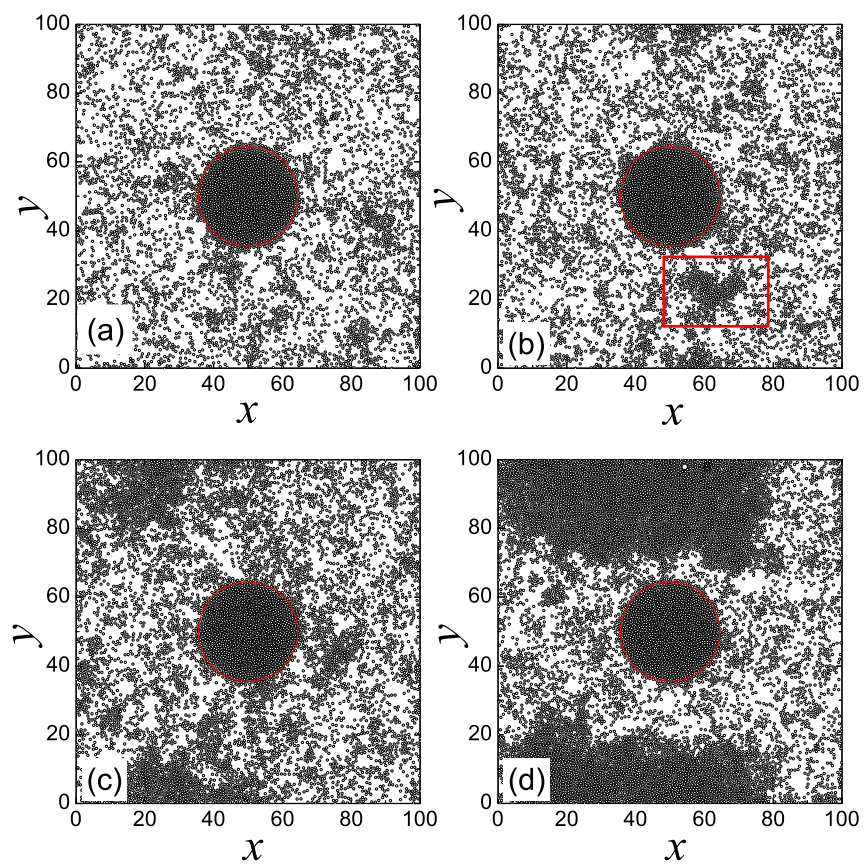

FIG. 6. (a)-(d) Formation of clusters outside the trap in "saturated" systems (when the trap is filled by microswimmers), for $D_{r}=$ $0.005, v_{0}=1.0, A=0.1$ and varying particle numbers: $N=6000$ (a), $N=6500$ (b), $N=8000$ (c), and $N=10000$ (d).

phase boundary for both the cases shown in Fig. 5: $N=100$ (a) and $N=500$ (b).

Finally, let us briefly discuss the case of high particle densities, i.e., when the number of microswimmers in the trap is saturated. In Fig. 6, typical patterns formed by microswimmers with $N=6000,6500,8000$, and 10000 are presented. When increasing the density of microswimmers above the critical value for the trap saturation, the free microswimmers outside the trap start to aggregate in clusters as shown in Fig. 6(b). This corresponds to the case when out of the total $N=6500$ microswimmers, approximately 1600 are trapped and 4900 are free. To characterize the collective behavior of random microswimmers outside the trap we define a packing fraction $\phi_{\text {out }}: \phi_{\text {out }}=N_{\text {out }} \pi(d / 2)^{2} /\left(S_{b}-S_{p}\right)$, where $N_{\text {out }}$ is the number of microswimmers outside the trap, $S_{b}$ is the area of the simulation box, and $S_{p}$ is the area of the parabolic trap. In particular, for $N=6500$ the packing fraction of free microswimmers is $\phi_{\text {out }} \approx 0.4$. This is consistent with the results known from literature that the critical packing fraction is $\phi_{\text {out }} \approx 0.4$ for the phase separation of gas and solidlike phases [23,31,32]. Further observations reveal a specific behavior, quite different from the one reported in the literature on the formation of clusters or living crystals. Thus, we observe a clear indication of the formation of clusters [Fig. 6(c)] and their further percolation into a solid [(Fig. 6(d)] near the boundaries of the simulation box rather than near the trap. This effective repulsion of the clusters outside the trap from the cluster confined inside the trap can be explained by an increased mobility of the microswimmers near the boundary of the trap where microswimmers repeatedly enter and leave the trap. Indeed, only the particles that possess sufficient velocity in the direction normal to the boundary of the trap are able 
to leave the trap. Leaving the trap, these higher mobility particles form a "high mobility" region outside the trap near its boundary (this follows from our calculations of the mobility of the particles which are not presented here) where the formation of clusters is less likely.

\section{v. CONCLUSIONS}

Using numerical simulations and analytical estimates based on a single-particle model, we analyzed the behavior and the formation of self-organized structures by artificial microswimmers (e.g., self-propelled Janus particles) in the presence of a weak attractive potential, or trap. We revealed new patterns, or phases, specific for the system of confined self-propelled particles, namely, self-organized shells and close-packed circular clusters. Unlike the living crystals, described in the literature, with shapes and structures varying in time (or even dissolving and re-forming again), the revealed patterns display striking dynamical stability. Furthermore, their shape, size, and the transitions between the different phases can be controlled by a number of system parameters such as the strength of the potential trap and the self-velocity of the microswimmers. In turn, the latter can be controlled by the intensity of the external light source (fueling Janus microswimmers). On the other hand, the strength of the trap can be monitored by the intensity of the laser or other source of the attractive trap. The results obtained here provide new insights into the dynamics of active particles (microswimmers) and could be useful for an understanding of the collective behavior of living active systems. Our predictions can be readily verified in experiment with either artificial microswimmers (Janus particles) or with active living systems (bacteria, sperm, or other active microswimmers) confined in a parabolic trap.

\section{ACKNOWLEDGMENTS}

This work was supported by the "Odysseus" Program of the Flemish Government and the Flemish Research Foundation (FWO-Vl) (Belgium), the Flemish Research Foundation (through Projects No. G.0115.12N, No. G.0119.12N, No. G.0122.12N, and No. G.0429.15N), and the Research Fund of the University of Antwerp. W.Y. acknowledges the support from the National Natural Science Foundation of China under Grants No. 11204199 and No. 51135007, the China Scholarship Council, the 131 project and the Program for the Outstanding Innovative Teams of Higher Learning Institutions of Shanxi, and a project under Grant No. 2016-096 by Shanxi Scholarship Council of China.
[1] R. Dreyfus, J. Baudry, M. L. Roper, M. Fermigier, H. A. Stone, and J. Bibette, Nature (London) 437, 862 (2005).

[2] G. A. Ozin, I. Manners, S. Fournier-Bidoz, and A. Arsenault, Adv. Mater. 17, 3011 (2005).

[3] J. Elgeti, R. G. Winkler, and G. Gompper, Rep. Prog. Phys. 78, 056601 (2015).

[4] N. C. Darnton, L. Turner, S. Rojevsky, and H. C. Berg, J. Bacteriol. 189, 1756 (2007).

[5] E. Lauga and R. E. Goldstein, Phys. Today 65(9), 30 (2012).

[6] K. Drescher, K. C. Leptos, I. Tuval, T. Ishikawa, T. J. Pedley, and R. E. Goldstein, Phys. Rev. Lett. 102, 168101 (2009).

[7] I. Buttinoni, G. Volpe, F. Kummel, G. Volpe, and C. Bechinger, J. Phys.: Condens. Matter 24, 284129 (2012).

[8] H.-R. Jiang, N. Yoshinaga, and M. Sano, Phys. Rev. Lett. 105, 268302 (2010).

[9] S. Fournier-Bidoz, A. Arsenault, I. Manners, and G. A. Ozin, Chem. Commun. 4, 441 (2005).

[10] I. Llopis and I. Pagonabarraga, Eur. Phys. J. E 26, 103 (2008).

[11] S. Sengupta, M. E. Ibele, and A. Sen, Angew. Chem., Int. Ed. 51, 8434 (2012).

[12] S. van Teeffelen and H. Lowen, Phys. Rev. E 78, 020101(R) (2008).

[13] J. R. Howse, R. A. L. Jones, A. J. Ryan, T. Gough, R. Vafabakhsh, and R. Golestanian, Phys. Rev. Lett. 99, 048102 (2007).

[14] P. K. Ghosh, V. R. Misko, F. Marchesoni, and F. Nori, Phys. Rev. Lett. 110, 268301 (2013).

[15] C. Reichhardt and C. J. Olson Reichhardt, Phys. Rev. E 88, 062310 (2013).

[16] J. A. Drocco, C. J. Olson Reichhardt, and C. Reichhardt, Phys. Rev. E 85, 056102 (2012).
[17] G. Mahmud, C. J. Campbell, K. J. M. Bishop, Y. A. Komarova, O. Chaga, S. Soh, S. Huda, K. Kandere-Grzybowska, and B. A. Grzybowski, Nat. Phys. 5, 606 (2009).

[18] M. B. Wan, C. J. Olson Reichhardt, Z. Nussinov, and C. Reichhardt, Phys. Rev. Lett. 101, 018102 (2008).

[19] P. Galajda, J. Keymer, P. Chaikin, and R. Austin, J. Bacteriol. 189, 8704 (2007).

[20] H. Yu, A. Kopach, V. R. Misko, A. A. Vasylenko, F. Marchesoni, F. Nori, D. Makarov, L. Baraban, and G. Cuniberti, Small 12, 5882 (2016).

[21] V. Narayan, S. Ramaswamy, and N. Menon, Science 317, 105 (2007).

[22] J. Deseigne, O. Dauchot, and H. Chate, Phys. Rev. Lett. 105, 098001 (2010).

[23] Y. Fily and M. C. Marchetti, Phys. Rev. Lett. 108, 235702 (2012).

[24] I. Theurkauff, C. Cottin-Bizonne, J. Palacci, C. Ybert, and L. Bocquet, Phys. Rev. Lett. 108, 268303 (2012).

[25] F. D. C. Farrell, M. C. Marchetti, D. Marenduzzo, and J. Tailleur, Phys. Rev. Lett. 108, 248101 (2012).

[26] S. K. Das, S. A. Egorov, B. Trefz, P. Virnau, and K. Binder, Phys. Rev. Lett. 112, 198301 (2014).

[27] J. Stenhammar, D. Marenduzzo, R. J. Allena, and M. E. Catesa, Soft Matter 10, 1489 (2014).

[28] A. Costanzo, J. Elgeti, T. Auth, G. Gompper, and M. Ripoll, Europhys. Lett. 107, 36003 (2014).

[29] W. Yang, V. R. Misko, K. Nelissen, M. Kong, and F. M. Peeters, Soft Matter 8, 5175 (2012).

[30] I. O. Gotze and G. Gompper, Phys. Rev. E 84, 031404 (2011). 
[31] J. Palacci, S. Sacanna, A. P. Steinberg, D. J. Pine, and P. M. Chaikin, Science 339, 936 (2013).

[32] I. Buttinoni, J. Bialke, F. Kummel, H. Löwen, C. Bechinger, and T. Speck, Phys. Rev. Lett. 110, 238301 (2013).

[33] R. van Drongelen, A. Pal, C. P. Goodrich, and T. Idema, Phys. Rev. E 91, 032706 (2015).

[34] R. W. Nash, R. Adhikari, J. Tailleur, and M. E. Cates, Phys. Rev. Lett. 104, 258101 (2010).
[35] A. P. Solon, J. Stenhammar, R. Wittkowski, M. Kardar, Y. Kafri, M. E. Cates, and J. Tailleur, Phys. Rev. Lett. 114, 198301 (2015).

[36] S. C. Takatori, W. Yan, and J. F. Brady, Phys. Rev. Lett. 113, 028103 (2014).

[37] V. M. Bedanov and F. M. Peeters, Phys. Rev. B 49, 2667 (1994).

[38] A. P. Solon, M. E. Cates, and J. Tailleur, Eur. Phys. J.: Spec. Top. 224, 1231 (2015). 